\title{
Giant spin Hall angle in the Heusler alloy Weyl ferromagnet Co2MnGa
}

$\operatorname{AUTHOR}(S)$ :

Leiva, L.; Granville, S.; Zhang, Y.; Dushenko, S.; Shigematsu, E.; Shinjo, T.; Ohshima, R.; Ando, Y.; Shiraishi, M.

\section{CITATION:}

Leiva, L. ...[et al]. Giant spin Hall angle in the Heusler alloy Weyl ferromagnet Co2MnGa. Physical Review B 2021, 103(4): L041114.

\section{ISSUE DATE:}

2021-01-15

URL:

http://hdl.handle.net/2433/261215

RIGHT:

(C)2021 American Physical Society 


\title{
Giant spin Hall angle in the Heusler alloy Weyl ferromagnet $\mathrm{Co}_{2} \mathrm{MnGa}$
}

\author{
L. Leiva $\odot,{ }^{1}$ S. Granville, ${ }^{2,3}$ Y. Zhang $\odot,{ }^{2,3}$ S. Dushenko $\odot,{ }^{1,4,5}$ E. Shigematsu, ${ }^{1}$ T. Shinjo, ${ }^{1}$ R. Ohshima, ${ }^{1}$ \\ Y. Ando, ${ }^{1}$ and M. Shiraishi ${ }^{1}{ }^{1}$ \\ ${ }^{1}$ Department of Electronic Science and Engineering, Kyoto University, Kyoto 615-8510, Japan \\ ${ }^{2}$ Robinson Research Institute, Victoria University of Wellington, Wellington 6140, New Zealand \\ ${ }^{3}$ The MacDiarmid Institute for Advanced Materials and Nanotechnology, Wellington 6011, New Zealand \\ ${ }^{4}$ Institute for Research in Electronics and Applied Physics, University of Maryland, College Park, Maryland 20742, USA \\ ${ }^{5}$ Physical Measurements Laboratory, National Institute of Standards and Technology, Gaithersburg, Maryland 20899, USA
}

(Received 28 August 2020; accepted 8 January 2021; published 29 January 2021)

\begin{abstract}
Weyl semimetals are playing a major role in condensed-matter physics due to exotic topological properties, and their coexistence with ferromagnetism may lead to enhanced spin-related phenomena. Here, the inverse spin Hall effect (ISHE) in the ferromagnetic Weyl semimetal Heusler alloy $\mathrm{Co}_{2} \mathrm{MnGa}$ was investigated at room temperature by means of electrical spin injection in lateral spin valve structures. Spin transport properties such as spin polarization and spin diffusion length in this material were precisely extracted in order to estimate the spin Hall angle $\theta_{\mathrm{SH}}$, which was found to be $-0.19 \pm 0.04$ and is among the highest reported for a ferromagnet. Although this value is on the same order of magnitude of known heavy metals, the significantly higher resistivity of $\mathrm{Co}_{2} \mathrm{MnGa}$ implies an improvement on the magnitude of detection voltages, while its ferromagnetic nature allows controlling the intensity of SHE through the magnetization direction. It was also shown that Onsager's reciprocity does not hold for this system, which is in part attributable to a different spin-dependent Hall conductivity for spin-up and spin-down carriers.
\end{abstract}

DOI: 10.1103/PhysRevB.103.L041114

Weyl semimetals have been attracting significant attention since the discovery of a nonmagnetic Weyl material, TaAs [1], because of its band-crossing points that give rise to plenty of unique physical properties, such as the Fermi arc surface states, the chiral anomaly coming from the Nielsen-Ninomiya theorem [2] and monopole-like Berry curvature [3]. Furthermore, a prominent class of Weyl semimetals is Weyl magnetic materials such as the ferromagnetic Heusler alloy $\mathrm{Co}_{2} \mathrm{MnGa}$ [4,5] and antiferromagnetic $\mathrm{Mn}_{3} \mathrm{Sn}$ [6]. These two materials are playing pivotal roles in condensed-matter physics because of the recent discoveries of the gigantic anomalous Hall effect (AHE) [7,8], the magnetic spin Hall effect (a novel family of Hall effects) [9], and spin caloritronics phenomena such as the large anomalous Nernst effect [4,10]. Additionally, magnetic Heusler alloys have emerged as promising materials in the field of spintronics due to their either half-metallic or semimetallic nature, which would lead to a high spin polarization [11,12], as has been reported in Co-based full Heusler compounds $[13,14]$. Combining the remarkable spin transport properties of Heusler alloys with the unique band structure of Weyl semimetals may lead to new exotic phenomena for topologically driven spintronic applications.

In the quest for these novel phenomena, an old acquaintance has emerged, the spin Hall effect (SHE). Together with its reciprocal version, the inverse spin Hall effect (ISHE), these two are utilized as essential methods for the generation and detection of pure spin currents in spintronics devices since they enable the conversion of a charge current into a transversal spin current and vice versa [15]. These spin-orbit coupling phenomena have been widely observed in many nonmagnetic heavy metals (HMs) [16-20], but it is thought that replacing a HM with a ferromagnet (FM) offers potential advantages such as precise control of the spin current through the magnetization direction, which could be applied to spin-transfer torque devices [21]. However, measurements on only a few ferromagnetic materials have been reported to date, most of them exhibiting spin Hall angles of just a few percentage points [22-28]. To overcome this limitation, materials exhibiting a large AHE are required since it is understood that both the AHE and the SHE are driven by the same intrinsic and extrinsic scattering mechanisms, related to the spin-orbit interaction $[29,30]$. The large Berry curvature distribution around the Fermi level in $\mathrm{Co}_{2} \mathrm{MnGa}$, thought to be responsible for its large AHE [31], and $a b$ initio calculations that predict a strong intrinsic spin Hall effect in other Weyl semimetals [32] indicate $\mathrm{Co}_{2} \mathrm{MnGa}$ is a strong candidate to observe large spin Hall voltages. In addition, significant spin polarization [33,34], strong resistance to oxidation [7], and a higher resistivity than conventional metals [35] suggest that $\mathrm{Co}_{2} \mathrm{MnGa}$ is a suitable platform to study the spin transport by means of electrical spin injection. In this work, the ISHE of $\mathrm{Co}_{2} \mathrm{MnGa}$ is investigated, wherein a giant spin Hall angle of $-0.19 \pm 0.04$ is found, which is among the highest reported for a FM so far.

The samples consisted of lateral spin valve (LSV) structures that were fabricated starting from 30-nm-thick films of $\mathrm{Co}_{2} \mathrm{MnGa}$ epitaxially grown on a (001)-oriented $\mathrm{MgO}$ single-crystal substrate. An x-ray diffraction (XRD) $\theta-2 \theta$ 


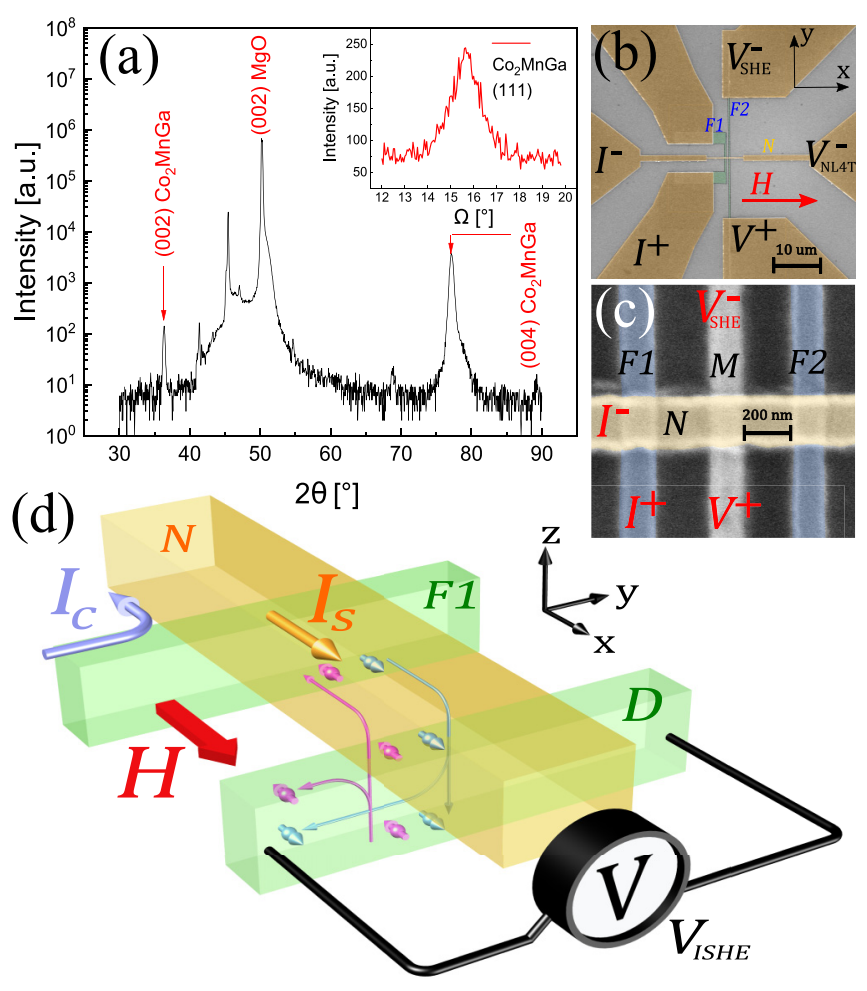

FIG. 1. (a) XRD pattern obtained for an out-of-plane $\theta-2 \theta$ scan of a sample consisting of a 30 -nm-thick $\mathrm{Co}_{2} \mathrm{MnGa}$ film grown on a single-crystal $\mathrm{MgO}$ substrate. Only the reflections due to the main x-ray source $\left(\mathrm{Co} K_{\alpha}\right)$ are indexed. The inset shows the rocking curve for the (111) peak. (b) Scanning electron microscope (SEM) image of a two-wire LSV. In an ISHE/SHE measurement, the magnetic field $H$ is applied parallel to the copper channel (color shown for clarity). (c) SEM image of a close view of the electrodes in a LSV with an absorption middle wire (color shown for clarity). (d) Schematics of the nonlocal ISHE measurement. A charge current $I_{c}$ is injected from $F 1$ to $N$, producing spin accumulation at the $F 1 / N$ interface, and a consequent spin current $I_{S}$ diffuses along $N$. Part of this spin current is absorbed by the detector $D$ in the negative $z$ direction. Then, a charge current in the positive $y$ direction is induced in $D$ due to the inverse spin Hall effect, and a voltage can be measured in the equilibrium open-circuit condition.

(out-of-plane) scan was performed to check the crystal structure in the films. The presence of (002) and (004) peaks of $\mathrm{Co}_{2} \mathrm{MnGa}$ confirms $B 2$ ordering in the thin films, as shown in Fig. 1(a), meanwhile the presence of a (111) peak, as verified by the rocking curve in the inset, confirms the $L 2_{1}$ ordering in the samples. The FM electrodes were patterned on the submicron scale using conventional electron-beam lithography and Ar ion milling techniques. Two types of devices were fabricated, one with two parallel FM electrodes [Fig. 1(b)] with various channel lengths $L$ (edge-to-edge separation) and one with three electrodes [Fig. 1(c)] with a fixed distance of $600 \mathrm{~nm}$ between $F 1$ and $F 2$ and various widths of the middle wire $M$ in order to estimate the spin resistance through the absorption technique [19,26,3640]. Finally, copper channels perpendicularly connecting the ferromagnetic electrodes, as well as the macroscopic connection pads, were patterned using electron-beam lithography and thermal evaporation techniques. It is worth mentioning that, in order to obtain Ohmic transparent interfaces, lowacceleration-voltage in situ $\mathrm{Ar}$ ion milling was performed prior to copper deposition. $\mathrm{Cu}$ was selected for the nonmagnetic channel because of its long spin diffusion length and long spin relaxation time [41], which make it a typical material for nonlocal signal measurements. For reference, devices with the same geometry but with Permalloy (Py) ferromagnetic electrodes were fabricated on a thermally oxidized Si substrate. In some of the two-wire Py devices, an absorption middle wire of $\mathrm{Pt}$ was deposited as a reference nonmagnetic material, as shown in the scanning electron microscope image in Fig. 1(c). All the transport measurements were performed at room temperature in a commercial physical property measurement system using a DC technique that consists of averaging the absolute value of the voltage measured with positive and negative DC currents, which is equivalent to an AC lock-in technique [42]. Further details of the fabrication procedure, as well as testing of the transparent interfaces and the determination of the spin diffusion length of $\mathrm{Co}_{2} \mathrm{MnGa}$, are presented in the Supplemental Material in Secs. A, B, and C [43].

Figure 1(d) shows the scheme of a typical ISHE measurement setup in a LSV. An electric current $I_{c}$ is injected from the $F 1$ electrode into the left side of the $N$ channel [terminals $I^{+}$and $I^{-}$in Figs. 1(b) and 1(c)], producing spin accumulation at the $F 1 / N$ interface that induces a diffusive pure spin current along the $N$ wire [44]. Part of the spin current is then absorbed vertically (negative $z$ direction) in a detection electrode $D$ ( $D$ can be $F 2$ or $M$ depending on the device). Since the spin orientation $\hat{s}$ of the conduction electrons is given by the magnetization of $F 1$, which is fixed by the external magnetic field applied along the $N$ channel, a charge current density $\boldsymbol{j}_{\boldsymbol{c}}$ given by $\boldsymbol{j}_{\boldsymbol{c}}=\theta_{S H} \hat{\boldsymbol{s}} \times \boldsymbol{j}_{\boldsymbol{s}}$ [45] (where $\theta_{S H}$ is the spin Hall angle and $\boldsymbol{j}_{\boldsymbol{s}}$ is the spin current density) is generated along the $D$ electrode length. In the equilibrium state in the open-circuit condition, a voltage $V_{\mathrm{ISHE}}$ is generated to suppress the charge current in $D$, measured between terminals $V^{+}$and $V_{\mathrm{SHE}}^{-}$. When the magnetization of the injection electrode is switched by sweeping the external magnetic field, the sign of the generated voltage is also expected to switch, as shown in the ISHE-labeled measurements in Fig. 2(c) for $\mathrm{Co}_{2} \mathrm{MnGa}$ and Fig. 2(d) for Pt and Py as detector electrodes, where $R_{\mathrm{ISHE}}=V_{\mathrm{ISHE}} / I_{c}$. The difference between the saturation values of $R_{\mathrm{ISHE}}$ for positive and negative fields is defined as $2 \Delta R_{\mathrm{ISHE}}$. Reciprocally, if the probe setup is inverted, by exchanging $V^{+}$with $I^{+}$and $V_{\mathrm{SHE}}^{-}$with $I^{-}$, a charge current along $D$ will inject a pure spin current into the $D / N$ interface by means of the (direct) SHE. This spin current will diffuse again across the $N$ channel to be detected as a nonlocal voltage $V_{\text {SHE }}$ between electrodes $F 1$ and $N$. The SHE setup nonlocal resistance $R_{\mathrm{SHE}}$ (defined as $V_{\mathrm{SHE}} / I_{c}$ ) is also shown in the SHE-labeled measurements in Fig. 2(c) for $\mathrm{Co}_{2} \mathrm{MnGa}$ and Fig. 2(d) for Pt and Py, where $2 \Delta R_{\mathrm{SHE}}$ can be compared with $2 \Delta R_{\text {ISHE }}$. Note that the magnitudes of ISHE and SHE signals for $\mathrm{Co}_{2} \mathrm{MnGa}$ are not the same, which is discussed later, and they are substantially large, roughly 20 times greater than for Pt and Py in very similar geometries. Anisotropic magnetoresistance (AMR) curves for the injection electrode $F 1$ are shown to prove that the saturation of the ISHE and SHE signals corresponds to the saturation of 
(a)

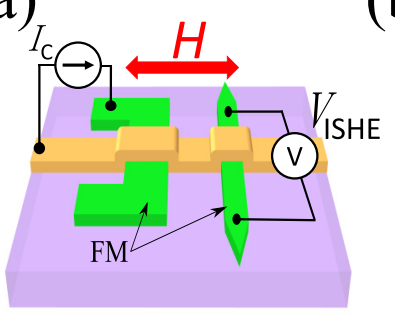

(b)

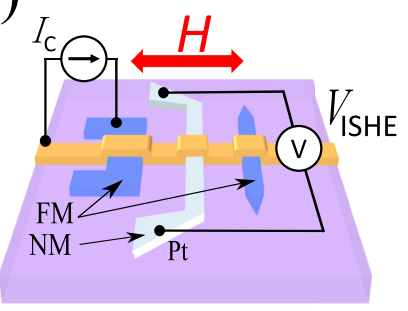

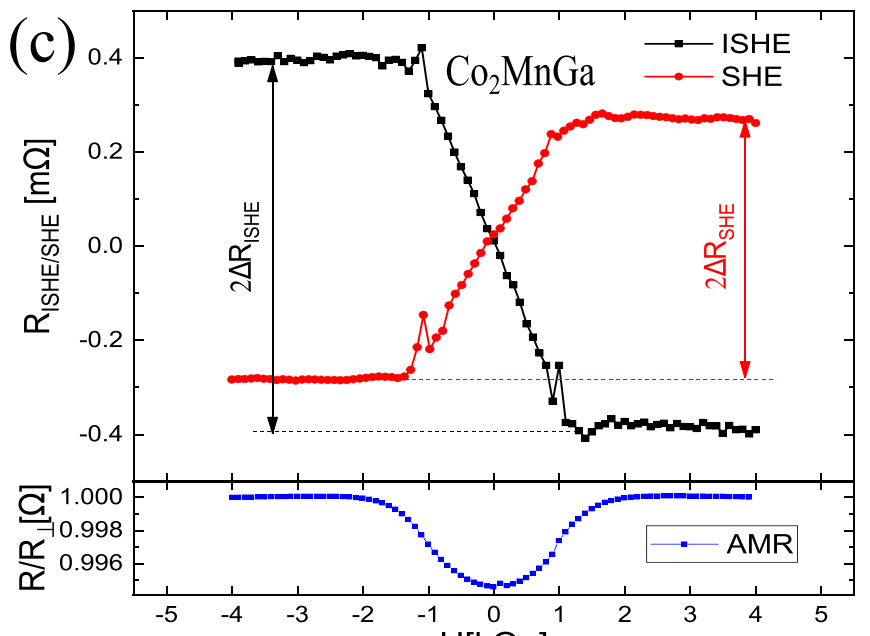

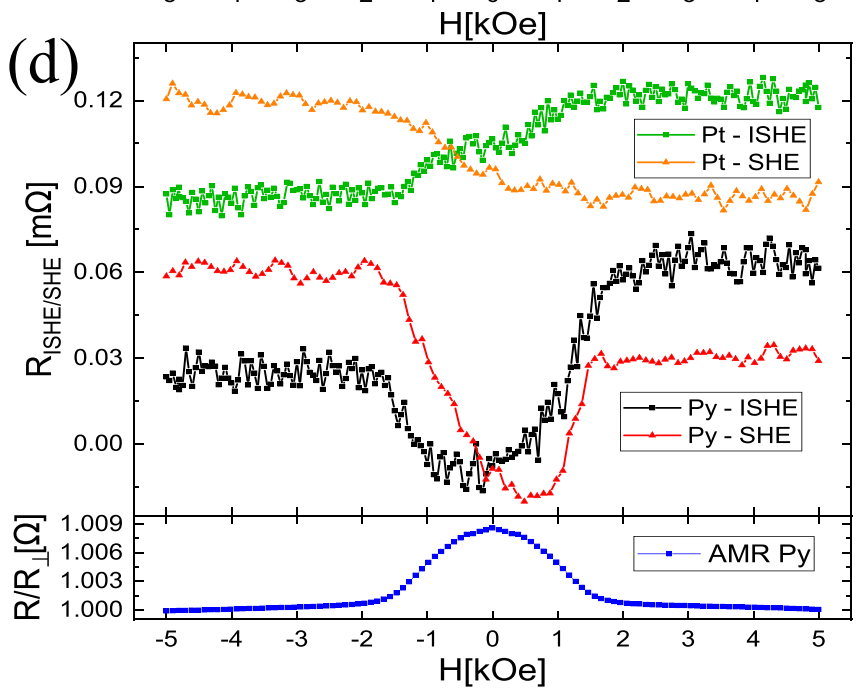

FIG. 2. (a) Schematics of the ISHE setup for a ferromagnetic detector. In this kind of sample both FM electrodes are made of the same material, either $\mathrm{Co}_{2} \mathrm{MnGa}$ or Py. For the SHE setup, the current source and voltmeter are exchanged, preserving the polarity. (b) Schematics of the ISHE setup for a nonmagnetic detector. In this case Py was used for FM electrodes, but one of them is not used in this measurement. The SHE setup is obtained by exchanging the current source with the voltmeter while preserving the polarity. (c) Nonlocal resistance of the ISHE setup and of its reciprocal (direct) SHE setup for $\mathrm{Co}_{2} \mathrm{MnGa}$, measured as a function of in-plane external magnetic field $H$ at room temperature. The bottom panel shows a typical AMR curve for $\mathrm{Co}_{2} \mathrm{MnGa}$ electrode $F 1$. (d) Equivalent nonlocal ISHE and SHE resistances measured for Pt and Py at room temperature. The bottom panel shows the AMR curve for electrode $F 1$ made of Py in both cases.

the magnetization of $F 1$, where it is worth noting that the AMR of $\mathrm{Co}_{2} \mathrm{MnGa}$ was found to be negative, as previously reported [46].

For the ISHE measurement setup, the ISHE resistance $\Delta R_{\text {ISHE }}$ can be expressed as $[37,45]$

$$
\Delta R_{\mathrm{ISHE}}=\frac{\left\langle I_{s}\right\rangle}{I_{c}} \theta_{S H} \frac{x}{W_{D}},
$$

where $\left\langle I_{S}\right\rangle$ is the spatial average of the absorbed spin current along the $z$ direction, $W_{D}$ is the width of the detector wire, and $x$ is the shunting factor that takes into account that part of the generated charge current is being shunted by the copper wire. By applying a one-dimensional spin diffusion model [47] for transparent interfaces and considering $t_{D} \gg \lambda_{D}$, where $t_{D}$ is the thickness of the detector electrode and $\lambda_{D}$ is its spin diffusion length, $\Delta R_{\mathrm{ISHE}}$ can be written as

$$
\Delta R_{\mathrm{ISHE}}^{2 W}=\frac{\theta_{S H} x W_{N}}{t_{F}} \frac{\left(1-\alpha_{F}^{2}\right) 2 \alpha_{F} R_{F}^{2} R_{N} e^{-L / \lambda_{N}}}{\left(2 R_{F}+R_{N}\right)^{2}-R_{N}^{2} e^{-2 L / \lambda_{N}}}
$$

for a two-wire LSV using $F 2$ as the detector and

$$
\begin{aligned}
\Delta & R_{\mathrm{ISHE}}^{3 W} \\
= & \frac{\theta_{S H} x W_{N}\left(1-\alpha_{M}^{2}\right)}{t_{M}} \\
& \times \frac{2 \alpha_{F} R_{F} R_{M} R_{N} e^{-L / 2 \lambda_{N}}}{\left(2 R_{F}+R_{N}\right)\left(2 R_{M}+R_{N}\right)-\left(2 R_{M}-R_{N}\right) R_{N} e^{-L / \lambda_{N}}}
\end{aligned}
$$

for a three-wire LSV using $M$ as detector, where $W_{N}$ is the width of the $N$ wire, $\lambda_{N}$ is the spin diffusion length of $\mathrm{Cu}$, $\alpha_{F}$ and $\alpha_{M}$ are the spin polarizations of the ferromagnetic electrodes and the middle wire $M$ material, respectively, and $R_{N}, R_{F}$, and $R_{M}$ are, respectively, the spin resistances of the $N$, $F 1$ (considered to be the same as $F 2$ ), and $M$ wires, defined in Ref. [43]. For Eq. (3) it was considered that the middle wire $M$ was located at the middle of the gap distance $L$ between the $F 1$ and $F 2$ electrodes.

In the case when a FM material is used as a detector electrode, $\Delta R_{\mathrm{ISHE}}$ can be written in terms of the conventional nonlocal-four-terminal (NL4T) resistance $\Delta R_{\mathrm{NL} 4 \mathrm{~T}}$, which can be measured by connecting the $V_{\mathrm{NL} 4 \mathrm{~T}}^{-}$terminal instead of $V_{\text {SHE }}^{-}$in Fig. 1(b) and sweeping the external magnetic field in a direction parallel to the ferromagnetic electrodes length. A typical NL4T signal is shown in the inset of Fig. 3(a), where two different voltage levels are measured depending on whether the relative configuration of the magnetization of the ferromagnetic electrodes is parallel or antiparallel. The difference between these two voltages, normalized by the injection current, define $\Delta R_{\mathrm{NL} 4 \mathrm{~T}}$, which is a direct measure of the amount of spin current being absorbed by the detection wire. Then, Eqs. (2) and (3) are simplified to

$$
\Delta R_{\mathrm{ISHE}}=\frac{\theta_{S H} x W_{N}}{t_{D}} \frac{\left(1-\alpha_{D}^{2}\right)}{2 \alpha_{D}} \Delta R_{\mathrm{NL} 4 \mathrm{~T}},
$$

where the only unknown spin transport parameter is the spin polarization of the detector electrode $\alpha_{D}$. In order to determine $\alpha_{D}, \Delta R_{\mathrm{NL} 4 \mathrm{~T}}$ was measured in two-wire LSVs for several 

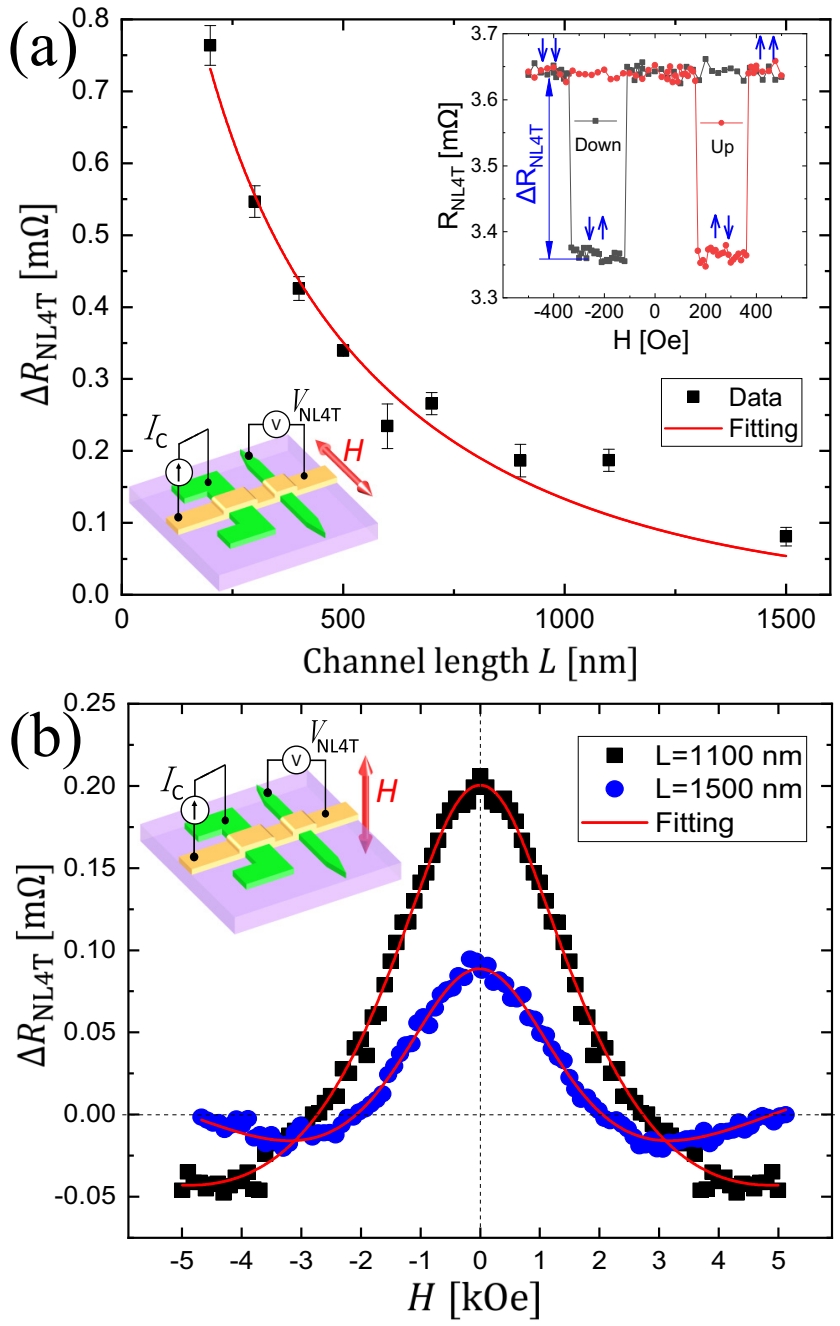

FIG. 3. (a) Channel length dependence of the nonlocal signal measured for several LSV devices at room temperature. The inset shows how $\Delta R_{\mathrm{NL} 4 \mathrm{~T}}$ is determined in an example of the acquired spin signal as a function of in-plane longitudinal magnetic field. (b) Hanle effect nonlocal signal as a function of the external out-of-plane magnetic field for two different channel lengths. A simultaneous fitting with common parameters is shown by the solid red line. In both panels, insets show a schematic of the measurement setup in each case.

different channel lengths $L$. This gap dependence is shown in Fig. 3(a), where the data are fitted according to the equation [47]

$$
\Delta R_{\mathrm{NL} 4 \mathrm{~T}}=\frac{4 \alpha_{F}^{2} R_{F}^{2} R_{N} e^{-L / \lambda_{N}}}{\left(2 R_{F}+R_{N}\right)^{2}-R_{N}^{2} e^{-2 L / \lambda_{N}}},
$$

where the only three unknown parameters are $\alpha_{F}, \lambda_{N}$, and $\lambda_{F}$, the spin diffusion length of the FM. Since $\alpha_{F}$ and $\lambda_{F}$ cannot be extracted independently, additional spin absorption measurements were performed to determine $\lambda_{F}$ self-consistently, yielding $\lambda_{F}=(3.1 \pm 1.1) \mathrm{nm}$ for $\mathrm{Co}_{2} \mathrm{MnGa}$ [43]. The spin polarization $\alpha_{F}$ was also determined via Hanle effect nonlocal measurements, which have the same geometry and sample structure as in the NL4T setup except for an out-of-plane external magnetic field instead of in plane. The data for two different channel lengths is shown in Fig. 3(b), where the

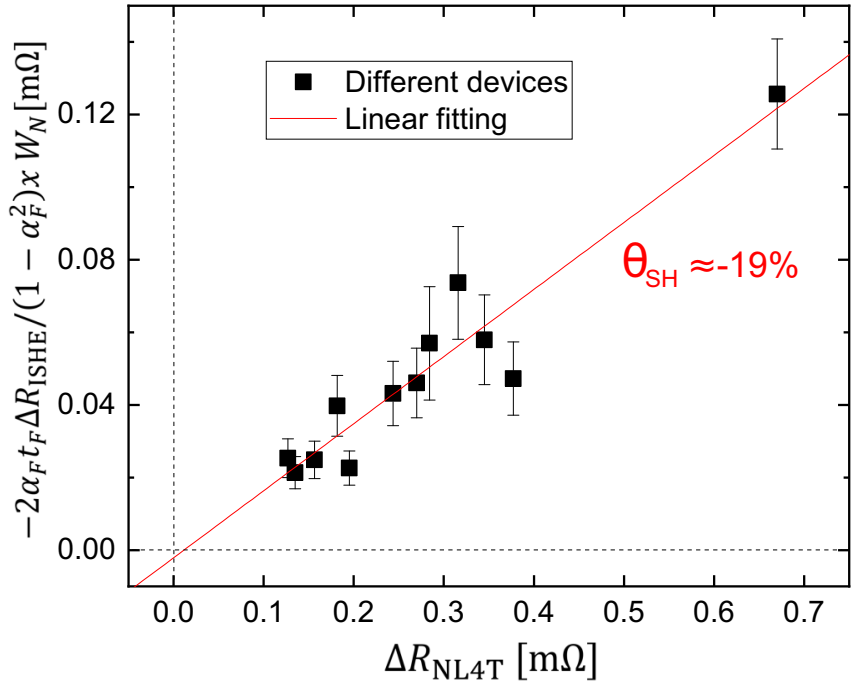

FIG. 4. Data for $-2 \alpha_{F} t_{F} \Delta R_{\mathrm{ISHE}} /\left(1-\alpha_{F}^{2}\right) W_{N} x$ as a function of $\Delta R_{\mathrm{NL} 4 \mathrm{~T}}$ obtained for $\mathrm{Co}_{2} \mathrm{MnGa}$ at room temperature for several devices. The spin Hall angle can be obtained from the slope of the linear fitting. Data points correspond to devices with different channel lengths $L$.

fitting was performed according to Eqs. (1), (2), and (3) in Ref. [48] for the case of transparent interfaces to consistently obtain $\alpha_{F}=0.15 \pm 0.03$.

The only unknown parameter left to estimate $\theta_{\mathrm{SH}}$ is the shunting factor $x$, which was determined experimentally with devices specially designed to that end, as detailed in Sec. D of the Supplemental Material [43]. Finally, a linear fitting, shown in Fig. 4, can be performed from the linear relation between $\Delta R_{\mathrm{NL} 4 \mathrm{~T}}$ and $\Delta R_{\mathrm{ISHE}}$ [Eq. (4)] to extract a large spin Hall angle $\theta_{\mathrm{SH}}=(-19 \pm 4) \%$, which is simply ascribable to the sizable Berry curvature as aforementioned.

According to Onsager's reciprocal relation [49], the resistances obtained for the SHE setup and ISHE setup should be the same, as is the case for the Py and Pt control samples in Fig. 2(b). This was first experimentally demonstrated by Kimura et al. [50] for the case of Pt and later verified for many other materials $[26,37,38]$. However, it was not the case for $\mathrm{Co}_{2} \mathrm{MnGa}$ samples, where ISHE and SHE signals are clearly different, as shown in Fig. 2(c). It is important to note that there is no effect of the geometry of the devices on whether the reciprocity holds or not, as verified in the control experiments detailed in Sec. H of the Supplemental Material [43].

While the origin of the reciprocity is well understood for nonmagnetic materials [51], it is still unclear why the relation should hold in FM systems since there is a breaking of the time-reversal symmetry. Furthermore, recent advances in the field suggest that nonreciprocal transport can exist in chiral materials such as Weyl semimetals [52].

In the case of $\mathrm{Co}_{2} \mathrm{MnGa}$, the nonreciprocity could be explained through a phenomenological picture of the Hall phenomena by introducing the spin-dependent spin Hall angles $\theta_{\uparrow}=\sigma_{x y}^{\uparrow} / \sigma_{x x}^{\uparrow}$ and $\theta_{\downarrow}=\sigma_{x y}^{\downarrow} / \sigma_{x x}^{\downarrow}$ and the polarization of the spin Hall angle $p_{\mathrm{SH}}$ through the relations $\theta_{\mathrm{SH}}=\left(\theta_{\uparrow}+\right.$ $\left.\theta_{\downarrow}\right) / 2$ and $p_{\mathrm{SH}}=\left(\theta_{\uparrow}-\theta_{\downarrow}\right) /\left(\theta_{\uparrow}+\theta_{\downarrow}\right)$, where $\sigma_{x y}^{s}(s=\uparrow, \downarrow)$ is the spin-dependent Hall conductivity and $\sigma_{x x}^{s}(s=\uparrow, \downarrow)$ 


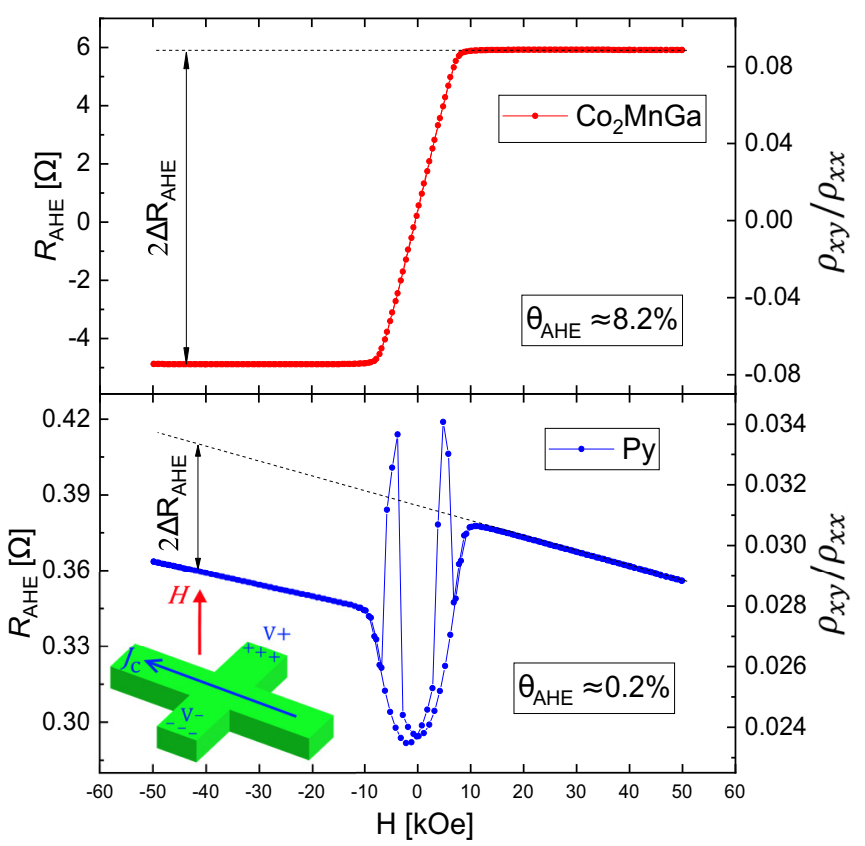

FIG. 5. Anomalous Hall resistance for $\mathrm{Co}_{2} \mathrm{MnGa}$ (top) and $\mathrm{Py}$ (bottom) measured at room temperature. The inset in the bottom panel shows a schematic of the measurement setup, where the magnetic field is applied out of plane in a Hall bar structure.

is the spin-dependent normal conductivity [26,34]. In the conventional case where $p_{\mathrm{SH}}$ is assumed to be zero, the anomalous Hall angle $\theta_{\mathrm{AHE}}$ is expected to be related to $\theta_{\mathrm{SH}}$ via the spin polarization $\alpha_{F}$ in the form $\theta_{\mathrm{AHE}}=\alpha_{F} \theta_{\mathrm{SH}}$ [22], as demonstrated for Py [26]. However, the values of $\theta_{\mathrm{AHE}}$ observed for $\mathrm{Co}_{2} \mathrm{MnGa}$ and Py have the same sign, as shown in Fig. 5, while $\theta_{\mathrm{SH}}$ exhibits different signs in both materials, as shown in Figs. 2(c) and 2(d). If a finite $p_{\mathrm{SH}}$ is consid- ered, the relation $\theta_{\mathrm{AHE}}=\left(\alpha_{F}+p_{\mathrm{SH}}\right) \theta_{\mathrm{SH}}$ should hold [43], indicating $p_{\mathrm{SH}}$ should be negative and $\left|p_{\mathrm{SH}}\right|>\alpha_{F}$. In addition, the relation between ISHE and SHE resistances obtained with the one-dimensional spin diffusion model [43],

$$
-\frac{\Delta R_{\mathrm{ISHE}}}{\Delta R_{\mathrm{SHE}}}=\frac{1-\alpha_{F}^{2}}{1+\alpha_{F} p_{\mathrm{SH}}}
$$

indicates $\left|\Delta R_{\mathrm{ISHE}}\right|>\left|\Delta R_{\mathrm{SHE}}\right|$, which is clear in Fig. 2(c). Refer to Sec. G of the Supplemental Material for a more detailed description of this spin-dependent spin Hall angle-based approach [43].

In conclusion, a direct and effective method to determine the spin Hall angle in ferromagnets was introduced to obtain a significantly large value of $\theta_{\mathrm{SH}}=(-19 \pm 4) \%$ for the Heusler alloy $\mathrm{Co}_{2} \mathrm{MnGa}$, a Weyl semimetal. Combined with the ability to control the intensity of ISHE through the magnetization direction and the high resistivity of the compound [43], this result situates $\mathrm{Co}_{2} \mathrm{MnGa}$ as a robust platform for the detection and generation of spin currents in future spintronic devices. Furthermore, a lack of reciprocity between ISHE and SHE resistances was observed and attributed to a negative polarization of the spin Hall angle.

This research was supported in part by a Grant-in-Aid for Scientific Research from the Ministry of Education, Culture, Sports, Science and Technology (MEXT) of Japan (Innovative Area "Nano Spin Conversion Science" KAKENHI No. 26103003), Grant-in-Aid for Young Scientists (A) No. 16H06089, and Grant-in-Aid for Scientific Research (S) "Semiconductor Spincurrentronics" No. 16H06330. L.L. acknowledges support from a MEXT doctoral scholarship. S.G. acknowledges financial support from the New Zealand Science for Technological Innovation National Science Challenge. The MacDiarmid Institute is supported under the New Zealand Centres of Research Excellence Programme.
[1] B. Q. Lv, H. M. Weng, B. B. Fu, X. P. Wang, H. Miao, J. Ma, P. Richard, X. C. Huang, L. X. Zhao, G. F. Chen, Z. Fang, X. Dai, T. Qian, and H. Ding, Experimental Discovery of Weyl Semimetal TaAs, Phys. Rev. X 5, 031013 (2015).

[2] H. Nielsen and M. Ninomiya, The Adler-Bell-Jackiw anomaly and Weyl fermions in a crystal, Phys. Lett. B 130, 389 (1983).

[3] H. Yang, Y. Sun, Y. Zhang, W.-J. Shi, S. S. Parkin, and B. Yan, Topological Weyl semimetals in the chiral antiferromagnetic materials $\mathrm{Mn}_{3} \mathrm{Ge}$ and $\mathrm{Mn}_{3} \mathrm{Sn}$, New J. Phys. 19, 015008 (2017)

[4] A. Sakai, Y. P. Mizuta, A. A. Nugroho, R. Sihombing, T. Koretsune, M.-T. Suzuki, N. Takemori, R. Ishii, D. NishioHamane, R. Arita, P. Goswami, and S. Nakatsuji, Giant anomalous Nernst effect and quantum-critical scaling in a ferromagnetic semimetal, Nat. Phys. 14, 1119 (2018).

[5] G. Chang, S. Y. Xu, X. Zhou, S. M. Huang, B. Singh, B. Wang, I. Belopolski, J. Yin, S. Zhang, A. Bansil, H. Lin, and M. Z. Hasan, Topological Hopf and Chain Link Semimetal States and Their Application to $\mathrm{Co}_{2} \mathrm{MnGa}$, Phys. Rev. Lett. 119, 156401 (2017).
[6] T. Matsuda, N. Kanda, T. Higo, N. Armitage, S. Nakatsuji, and R. Matsunaga, Room-temperature terahertz anomalous Hall effect in Weyl antiferromagnet $\mathrm{Mn}_{3} \mathrm{Sn}$ thin films, Nat. Commun. 11, 909 (2020).

[7] B. M. Ludbrook, B. J. Ruck, and S. Granville, Perpendicular magnetic anisotropy in $\mathrm{Co}_{2} \mathrm{MnGa}$ and its anomalous Hall effect, Appl. Phys. Lett. 110, 062408 (2017).

[8] S. Nakatsuji, N. Kiyohara, and T. Higo, Large anomalous Hall effect in a non-collinear antiferromagnet at room temperature, Nature (London) 527, 212 (2015).

[9] M. Kimata, H. Chen, K. Kondou, S. Sugimoto, P. K. Muduli, M. Ikhlas, Y. Omori, T. Tomita, A. H. MacDonald, S. Nakatsuji, and Y. Otani, Magnetic and magnetic inverse spin Hall effects in a non-collinear antiferromagnet, Nature (London) 565, 627 (2019).

[10] H. Reichlova, R. Schlitz, S. Beckert, P. Swekis, A. Markou, Y.C. Chen, D. Kriegner, S. Fabretti, G. Hyeon Park, A. Niemann, S. Sudheendra, A. Thomas, K. Nielsch, C. Felser, and S. T. B. Goennenwein, Large anomalous Nernst effect in thin films of the Weyl semimetal $\mathrm{Co}_{2} \mathrm{MnGa}$, Appl. Phys. Lett. 113, 212405 (2018). 
[11] J. M. D. Coey and M. Venkatesan, Half-metallic ferromagnetism: Example of $\mathrm{CrO}_{2}$ (invited), J. Appl. Phys. 91, 8345 (2002).

[12] C. Felser and G. H. Fecher, Spintronics: From Materials to Devices (Springer, Dordrecht, Heidelberg, New York, London, 2013).

[13] T. Kimura, N. Hashimoto, S. Yamada, M. Miyao, and K. Hamaya, Room-temperature generation of giant pure spin currents using epitaxial $\mathrm{Co}_{2} \mathrm{FeSi}$ spin injectors, NPG Asia Mater. 4, e9 (2012).

[14] S. Shirotori, S. Hashimoto, M. Takagishi, Y. Kamiguchi, and H. Iwasaki, All-metallic nonlocal spin valves using polycrystalline $\mathrm{Co}_{2}(\mathrm{FeMn}) \mathrm{Si}$ Heusler alloy with large output, Appl. Phys. Express 8, 023103 (2015).

[15] J. Sinova, S. O. Valenzuela, J. Wunderlich, C. H. Back, and T. Jungwirth, Spin Hall effects, Rev. Mod. Phys. 87, 1213 (2015).

[16] E. Saitoh, M. Ueda, and H. Miyajima, Conversion of spin current into charge current at room temperature: Inverse spin-Hall effect, Appl. Phys. Lett. 88, 182509 (2006).

[17] H. L. Wang, C. H. Du, Y. Pu, R. Adur, P. C. Hammel, and F. Y. Yang, Scaling of Spin Hall Angle in 3d, 4d, and 5d Metals from $\mathrm{Y}_{3} \mathrm{Fe}_{5} \mathrm{O}_{12}$ /Metal Spin Pumping, Phys. Rev. Lett. 112, 197201 (2014).

[18] X. Tao, Q. Liu, B. Miao, R. Yu, Z. Feng, L. Sun, B. You, J. Du, K. Chen, S. Zhang, L. Zhang, Z. Yuan, D. Wu, and H. Ding, Self-consistent determination of spin Hall angle and spin diffusion length in $\mathrm{Pt}$ and $\mathrm{Pd}$ : The role of the interface spin loss, Sci. Adv. 4, eaat1670 (2018).

[19] M. Morota, Y. Niimi, K. Ohnishi, D. H. Wei, T. Tanaka, H. Kontani, T. Kimura, and Y. Otani, Indication of intrinsic spin Hall effect in $4 d$ and $5 d$ transition metals, Phys. Rev. B 83, 174405 (2011).

[20] O. Mosendz, J. E. Pearson, F. Y. Fradin, G. E. W. Bauer, S. D. Bader, and A. Hoffmann, Quantifying Spin Hall Angles from Spin Pumping: Experiments and Theory, Phys. Rev. Lett. 104, 046601 (2010).

[21] T. Taniguchi, J. Grollier, and M. D. Stiles, Spin-Transfer Torques Generated by the Anomalous Hall Effect and Anisotropic Magnetoresistance, Phys. Rev. Appl. 3, 044001 (2015).

[22] A. Tsukahara, Y. Ando, Y. Kitamura, H. Emoto, E. Shikoh, M. P. Delmo, T. Shinjo, and M. Shiraishi, Self-induced inverse spin Hall effect in permalloy at room temperature, Phys. Rev. B 89, 235317 (2014).

[23] B. F. Miao, S. Y. Huang, D. Qu, and C. L. Chien, Inverse Spin Hall Effect in a Ferromagnetic Metal, Phys. Rev. Lett. 111, 066602 (2013).

[24] T. Seki, K.-i. Uchida, T. Kikkawa, Z. Qiu, E. Saitoh, and K. Takanashi, Observation of inverse spin Hall effect in ferromagnetic FePt alloys using spin Seebeck effect, Appl. Phys. Lett. 107, 092401 (2015).

[25] T. Seki, S. Iihama, T. Taniguchi, and K. Takanashi, Large spin anomalous Hall effect in $l 1_{0}$-FePt: Symmetry and magnetization switching, Phys. Rev. B 100, 144427 (2019).

[26] Y. Omori, E. Sagasta, Y. Niimi, M. Gradhand, L. E. Hueso, F. Casanova, and Y. C. Otani, Relation between spin Hall effect and anomalous Hall effect in $3 d$ ferromagnetic metals, Phys. Rev. B 99, 014403 (2019).

[27] S. Iihama, T. Taniguchi, K. Yakushiji, A. Fukushima, Y. Shiota, S. Tsunegi, R. Hiramatsu, S. Yuasa, Y. Suzuki, and H. Kubota,
Spin-transfer torque induced by the spin anomalous Hall effect, Nat. Electron. 1, 120 (2018).

[28] T. Wimmer, B. Coester, S. Geprägs, R. Gross, S. T. Goennenwein, H. Huebl, and M. Althammer, Anomalous spin Hall angle of a metallic ferromagnet determined by a multiterminal spin injection/detection device, Appl. Phys. Lett. 115, 092404 (2019).

[29] B. Zimmermann, K. Chadova, D. Ködderitzsch, S. Blügel, H. Ebert, D. V. Fedorov, N. H. Long, P. Mavropoulos, I. Mertig, Y. Mokrousov, and M. Gradhand, Skew scattering in dilute ferromagnetic alloys, Phys. Rev. B 90, 220403(R) (2014).

[30] A. Hoffmann, Spin Hall effects in metals, IEEE Trans. Magn. 49, 5172 (2013).

[31] A. Markou, D. Kriegner, J. Gayles, L. Zhang, Y.-C. Chen, B. Ernst, Y.-H. Lai, W. Schnelle, Y.-H. Chu, Y. Sun, and C. Felser, Thickness dependence of the anomalous Hall effect in thin films of the topological semimetal $\mathrm{Co}_{2} \mathrm{MnGa}$, Phys. Rev. B 100, 054422 (2019).

[32] Y. Sun, Y. Zhang, C. Felser, and B. Yan, Strong Intrinsic Spin Hall Effect in the TaAs Family of Weyl Semimetals, Phys. Rev. Lett. 117, 146403 (2016).

[33] M. Kolbe, S. Chadov, E. A. Jorge, G. Schönhense, C. Felser, H. J. Elmers, M. Kläui, and M. Jourdan, Test of band structure calculations for Heusler compounds by spinresolved photoemission spectroscopy, Phys. Rev. B 86, 024422 (2012).

[34] J.-C. Tung and G.-Y. Guo, High spin polarization of the anomalous Hall current in Co-based Heusler compounds, New J. Phys. 15, 033014 (2013).

[35] S. Holmes and M. Pepper, Magnetic and electrical properties of $\mathrm{Co}_{2} \mathrm{MnGa}$ grown on GaAs (001), Appl. Phys. Lett. 81, 1651 (2002).

[36] P. Laczkowski, H. Jaffrès, W. Savero-Torres, J.-C. RojasSánchez, Y. Fu, N. Reyren, C. Deranlot, L. Notin, C. Beigné, J.-P. Attané, L. Vila, J.-M. George, and A. Marty, Evaluation of spin diffusion length of AuW alloys using spin absorption experiments in the limit of large spin-orbit interactions, Phys. Rev. B 92, 214405 (2015).

[37] Y. Niimi, M. Morota, D. H. Wei, C. Deranlot, M. Basletic, A. Hamzic, A. Fert, and Y. Otani, Extrinsic Spin Hall Effect Induced by Iridium Impurities in Copper, Phys. Rev. Lett. 106, 126601 (2011).

[38] Y. Niimi, Y. Kawanishi, D. H. Wei, C. Deranlot, H. X. Yang, M. Chshiev, T. Valet, A. Fert, and Y. Otani, Giant Spin Hall Effect Induced by Skew Scattering from Bismuth Impurities Inside Thin Film CuBi Alloys, Phys. Rev. Lett. 109, 156602 (2012)

[39] E. Sagasta, Y. Omori, M. Isasa, Y. Otani, L. E. Hueso, and F. Casanova, Spin diffusion length of permalloy using spin absorption in lateral spin valves, Appl. Phys. Lett. 111, 082407 (2017).

[40] G. Zahnd, L. Vila, V. T. Pham, M. Cosset-Cheneau, W. Lim, A. Brenac, P. Laczkowski, A. Marty, and J. P. Attané, Spin diffusion length and polarization of ferromagnetic metals measured by the spin-absorption technique in lateral spin valves, Phys. Rev. B 98, 174414 (2018).

[41] F. J. Jedema, M. S. Nijboer, A. T. Filip, and B. J. van Wees, Spin injection and spin accumulation in all-metal mesoscopic spin valves, Phys. Rev. B 67, 085319 (2003). 
[42] F. Casanova, A. Sharoni, M. Erekhinsky, and I. K. Schuller, Control of spin injection by direct current in lateral spin valves, Phys. Rev. B 79, 184415 (2009).

[43] See Supplemental Material at http://link.aps.org/supplemental/ 10.1103/PhysRevB.103.L041114 for details on sample preparation, determination of relevant parameters, characterization of magnetic electrodes, the angle dependence of the ISHE signal, and a discussion of the validity of reciprocal relations.

[44] S. O. Valenzuela, Nonlocal electronic spin detection, spin accumulation and the spin Hall effect, Int. J. Mod. Phys. B 23, 2413 (2009).

[45] S. Takahashi and S. Maekawa, Spin current, spin accumulation and spin Hall effect, Sci. Technol. Adv. Mater. 9, 014105 (2008).

[46] T. Sato, S. Kokado, S. Kosaka, T. Ishikawa, T. Ogawa, and M. Tsunoda, Large negative anisotropic magnetoresistance in $\mathrm{Co}_{2} \mathrm{MnGa}$ Heusler alloy epitaxial thin films, Appl. Phys. Lett. 113, 112407 (2018).
[47] S. Takahashi and S. Maekawa, Spin injection and detection in magnetic nanostructures, Phys. Rev. B 67, 052409 (2003).

[48] J.-C. Rojas Sánchez, P. Laczkowski, W. Savero Torres, M. Cubukcu, V. Nguyen, L. Notin, C. Beigné, C. Vergnaud, A. Marty, M. Jamet, L. Vila, and J. P. Attané, In-plane and out-ofplane spin precession in lateral spin-valves, Appl. Phys. Lett. 102, 132408 (2013).

[49] L. Onsager, Reciprocal relations in irreversible processes. II, Phys. Rev. 38, 2265 (1931).

[50] T. Kimura, Y. Otani, T. Sato, S. Takahashi, and S. Maekawa, Room-Temperature Reversible Spin Hall Effect, Phys. Rev. Lett. 98, 156601 (2007).

[51] P. Jacquod, R. S. Whitney, J. Meair, and M. Büttiker, Onsager relations in coupled electric, thermoelectric, and spin transport: The tenfold way, Phys. Rev. B 86, 155118 (2012).

[52] Y. Tokura and N. Nagaosa, Nonreciprocal responses from noncentrosymmetric quantum materials, Nat. Commun. 9, 3740 (2018). 